\title{
Nano-Bio Fusion Science Opened and Created with Plasmas
}

\author{
Rikizo HATAKEYAMA $^{1)}$ and Toshiro KANEKO ${ }^{1,2)}$ \\ ${ }^{1)}$ Department of Electronic Engineering, Tohoku University, Sendai 980-8579, Japan \\ ${ }^{2)}$ CREST/JST, Tokyo 102-0075, Japan
}

(Received 24 December 2010 / Accepted 4 February 2011)

\begin{abstract}
Recent years have brought about a rapid rise in research on applications in biological and medical fields including cell and tissue inactivation, sterilization and disinfection, blood coagulation, therapy, surgery, pharmaceuticals, biosensing, and biochips by exploiting low-pressure, atmospheric-pressure, and solution plasma processes. Many of the basic constituent materials of biological organisms are nanometer-scale in size, thus presenting the possibility of a growing field of science and technology focused on a fusion between "bio" and "nano" mechanisms; we are now in the germinal stage of a new era of next-generation applied research in the biological and medical applications of nanocarbons typified by fullerenes and carbon nanotubes. In this context, the authors have proposed and implemented research utilizing nanoscopic processes in advanced gas, liquid, and gas-liquid interfacial plasmas, directed toward the creation of synthetic materials and devices composed of nanocarbons, nanoparticles, and biomolecules. This research is positioned to advance the establishment of nano-bio fusion science opened and created with plasmas to construct next-generation nanobio and medical systems relating to nanobio-electronics devices, intracellular nanoengineering, and nanomedicine.
\end{abstract}

(c) 2011 The Japan Society of Plasma Science and Nuclear Fusion Research

Keywords: plasmas for nano-bio fusion, gas/liquid/interface, nanocarbon, nanoparticle, biomolecule

DOI: $10.1585 /$ pfr.6.1106011

\section{Introduction}

With the rising interest in the global environment and social welfare, biological and medical technologies and systems are expected to grow rapidly in importance. They will deal with fundamental elements such as DNA, peptides, proteins, and other biological polymers. At present, plasma processes are being actively applied in biological and medical research, which is based on direct mutual interaction between these elements and plasma media that offer a wealth of means for control of physical mechanisms involving electrons, ions, electric fields, heat, radiation, and chemical reactions involving oxygen, nitrogen, and other radicals. In these plasma processes, low-pressure, atmospheric-pressure, and solution plasmas generated by corona, glow, arc, and other electrical discharges are utilized for cell and tissue inactivation, sterilization, disinfection, and for blood coagulation, therapy, and surgery. Research is now in progress from the viewpoint of the creation of bio-originating and functionalinterface substances, materials, and devices towards pharmaceuticals, bio-material coatings, biosensing, biochips, targeting, imaging, drug delivery, and other fields of application.

New concepts, substances and materials, technologies, and systems will emerge in the light of the multifaceted opportunities implied in the proliferation of new technical terms prefixed by "nano" and "bio", in many fields. As many biological polymers and other constituent elements of cell life are nanometer-order in size, the trend has naturally arisen for the fusion of nanotechnology into biotechnology, and the new life-related scientific and technological research of nano-bio fusion is now in progress. For the authors, this has led to a research effort with the ultimate goal of creating a "plasma biotronics" for the construction of next-generation nanobio and medical systems involving nanobio-electronics devices, intracellular nanoengineering, and nanomedicine, through utilization of nanoscopic processes in advanced gas, liquid, and gasliquid interfacial plasmas (Fig. 1). It is, in short, directed toward the application of plasmas to open the way and create a new science, which we term "nano-bio fusion science". At present, the "nano" constituents consist mainly of carbon-allotrope nanocarbons, including fullerenes with their basically zero-dimensional structure $\left(\mathrm{C}_{60}\right)$ and carbon nanotubes (CNTs) with their one-dimensional structure, as well as gold and other metal nanoparticles, while the "bio" constituents consist mainly of the biological polymer DNA.

In the present report, we begin with an overview of the current state of plasma process biological and medical applications, proceed from there to a discussion of nextgeneration biological and medical applications of nanocarbons, and close with a description of the developmental pathway from plasma nanobio processes to plasma nanobio-electronics. 


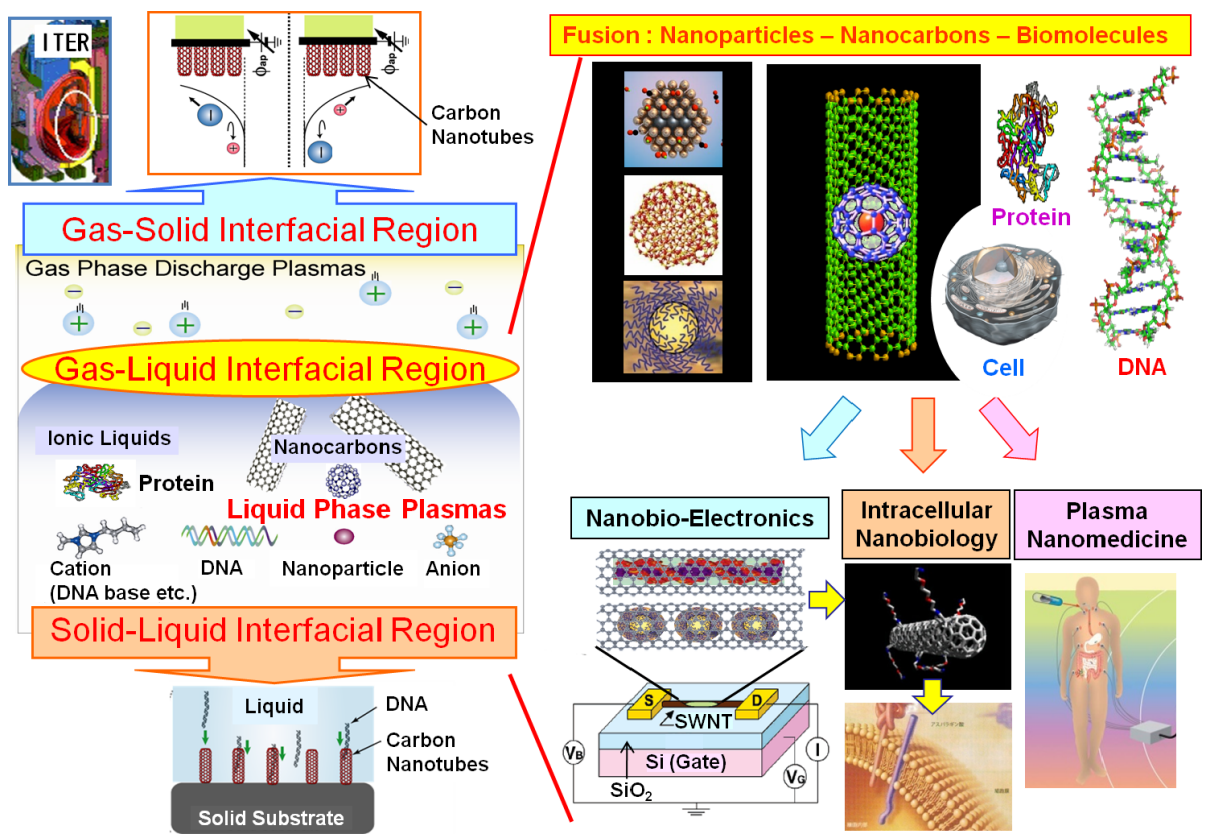

Fig. 1 Conception of nano-bio fusion science using solid-gas-liquid interfacial plasmas.

\section{Current State of Biological and Medical Plasma Process Applica- tions}

One important category of current plasma process applications is the formation of biomaterial coatings by DCthermal or RF-thermal plasma spraying, as medical materials for artificial hip joints and tooth roots [1]. In artificial hip joint clinical applications, the bioactive ceramic hydroxyapatite (HA), which bonds directly to bone, is applied as a coating between bone tissue and metal implant. It promotes bone conduction, maintains long-term interface stability, and enables attainment of high bonding strength by the formation of a HA/Ti gradient composite film. In this application DC plasma spraying may generate cytotoxic contamination due to electrode attrition, and RF plasma spraying is superior as it ensures a high-quality HA coating. The process is essentially one of simply using a plasma torch as the heat source, but plasma reactivity has drawn increasing interest in recent years as a means of surface modification. It has, for example, been reported that the HA/Ti composite film adhesion can be markedly increased by adding nitrogen as the working gas.

A second category of current applications is that of inactivation, sterilization, and disinfection. As a new process enabling high-speed and low-temperature sterilization without the use of harmful/hazardous substances, "cold plasma sterilization" has become a focus of strong interest. In early experiments for sterilization of Bacillus Atropheus by a low-pressure microwave-discharge afterglow plasma containing nitrogen and oxygen mixed gas, three different sterilization slopes were found in the plot of surviving micro-organisms vs. processing time [2], as shown

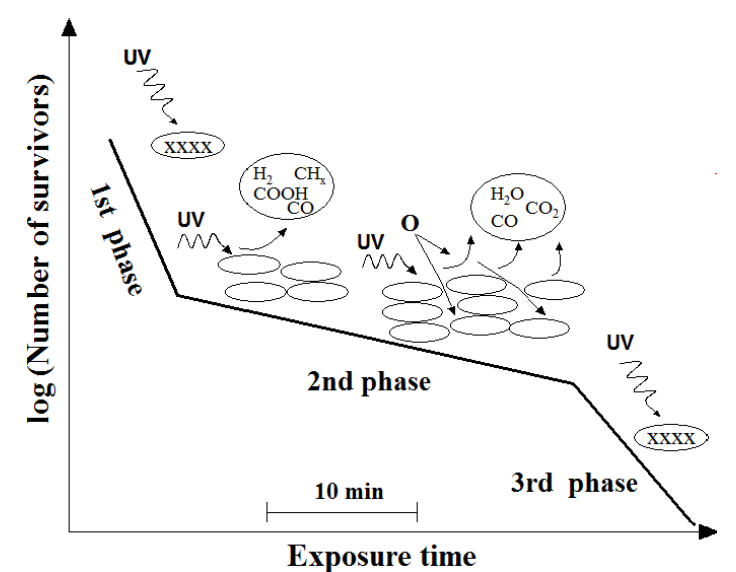

Fig. 2 Interpretive model of survival in sterilization experiments with afterglow microwave plasma.

schematically in Fig. 2. The initial sterilization slope (1st phase) is interpreted as representing inactivation of DNA by UV rays radiated from the plasma, the next slope (2nd phase) as representing a synergy between this effect and surface-layer bacteria etching by oxygen radicals, and the final slope (3rd phase) as representing renewed UV bacterial inactivation, in the exposed bacteria layer of the innermost regions. In this process, the bacteria inactivation time was long (30 40 min) and it was difficult to realize large-capacity equipments. The afterglow plasma was therefore replaced with a microwave-excited surface-wave oxygen and nitrogen plasma, as shown in Fig. 3, which was found to result in bacteria annihilation in $3 \sim 5 \mathrm{~min}$ and also showed the importance of the $\mathrm{OH}$ radical in addition to the synergy between oxygen radical etching and 


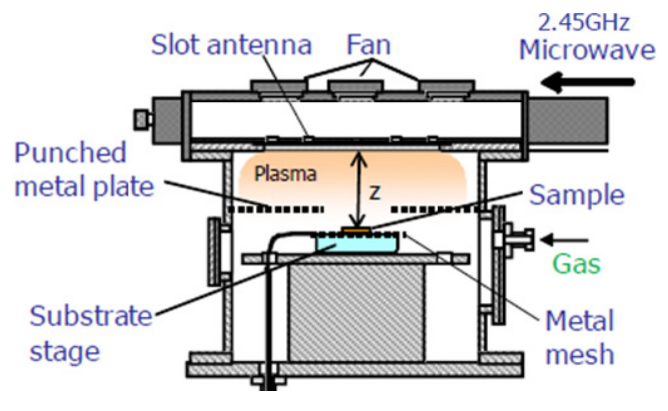

Fig. 3 Microwave-excited surface-wave plasma device for medical sterilization applications.

(a)

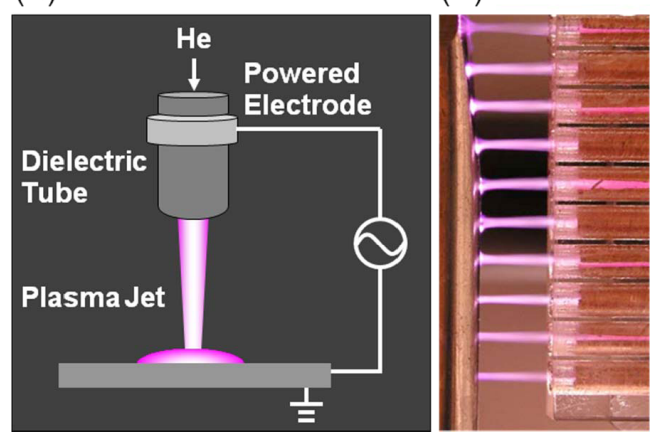

Fig. 4 (a) Cold atmospheric-pressure plasma jet and electrode configuration, and (b) 3D surface-treatment plasma jet array.

UV disinfection [3]. To meet the need to proceed from surface sterilization to three-dimensional sterilization, the low-pressure discharge was replaced by a high-pressure discharge. More specifically, the investigation proceeded to cold-sterilization experiments for the inside of medical instrument packaging containers, using the atmospheric dielectric barrier discharge $( \pm 2.5 \mathrm{kV}, 5 \mathrm{kHz})[4]$.

In the next series of investigations aiming to inactivate substances ranging from cells to biomolecules, the focus was shifted and advanced to the utilization of a "cold atmospheric plasma jet" (Fig. 4 (a)), a modification of the early plasma pencil, in which a plume was formed by spout from a small aperture [5]. These experiments were performed with protein BSA (neurodegenerative bovine serum albumin) as the model of a medical instrument contaminator, which was irradiated with an atmospheric $\mathrm{He}-\mathrm{O}_{2}$ mixedplasma jet ( $7 \mathrm{kHz}$ : sine wave $7.3 \mathrm{kV}$, pulse $4 \mathrm{kV})$, and it is reported that maximum inactivation was obtained with $\mathrm{O}_{2} / \mathrm{He}=0.5 \%$ and that biological molecule inactivation was also obtained in virulent tumor cells (G361). As shown in Fig. 4 (b), an atmospheric plasma jet array ( $30 \mathrm{kV}$, maximum 30 parallel channels) for three-dimensional surface treatment has also been developed, and spatial and temporal uniformity of the plasma jets has been observed, largely as the effect of a self-adjustment mechanism among

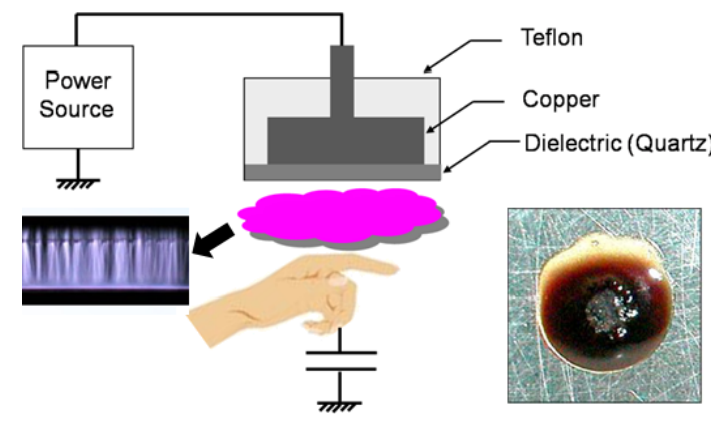

Fig. 5 Schematic of floating-electrode dielectric barrier discharge plasma system and photograph of blood coagulation and hemostasis.

individual jets facilitated by individualized ballast and possibly also by spatial redistribution of surface charges, with the jet array thus behaving like one integrated plasma with effective internal feedback among the individual jets [6].

In the application of plasma for coagulation, therapy, and surgery, a shift from the above-described remote jet plasma to "floating-electrode dielectric barrier discharge plasma" (Fig. 5), with the biological tissue itself as one electrode, marks an epochal advance [7]. The use of nonthermal room-temperature plasma, with free discharge in air, enables safe treatment of biological tissue in living animals and humans, making possible tissue sterilization and blood coagulation without thermally or chemically injuring the tissue. The gap current consists of moving electrons and ions, but it proceeds through the tissue largely as a displacement current and most of the energy from the plasma current thus dissipating in the gap. A few seconds of plasma irradiation in mice has been found to be sufficient for sterilization, with no apparent pain or fatigue even two weeks after the irradiation and no apparent tissue toxicity, and effective blood coagulation and hemostasis have also been confirmed (Fig. 5 insert). The first of these techniques brought to full development was the "argon plasma coagulation technique" (APC), for simultaneous tissue excision (deactivation) and hemostasis. It employs cold, nonequilibrium plasma produced by relatively high current with a $20 \mathrm{kHz}$ burst period and high-voltage insulation breakdown. Unlike laser therapy, it causes no cell ablation and no soot-forming carbonization, and it facilitates control of inactivation, coagulation, and drying all at a uniform depth and extending over a broad range. The direction of the plasma flow is that of the electric field rather than the Ar gas, and the regions of coagulation and bleeding are self-controlled by their differences in electric conductivity (with searching performed by peripheral scanning). APC is currently used clinically in combination with a flexible endoscope in internal medicine and ENT (ear, nose, and throat) therapies [8].

Development is now in progress for an instrument which utilizes a multipolar circuit configuration to reduce 
(a)

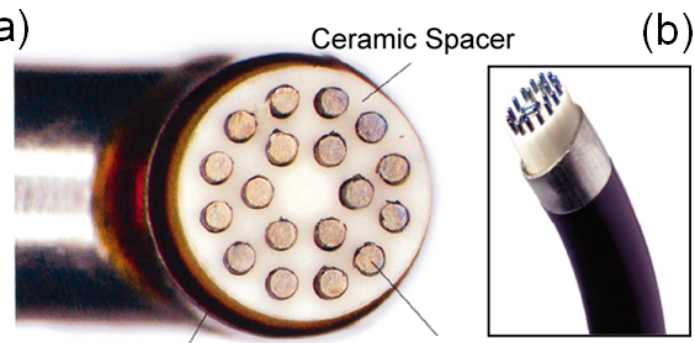

Stainless Steel Titanium Electrode Current Return Electrode

(c)

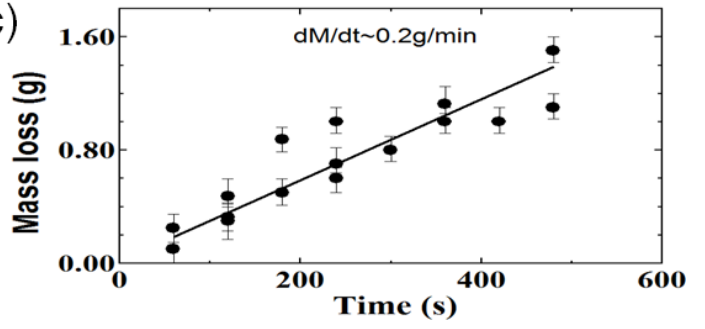

Fig. 6 (a) Multi-electrode solution-discharge plasma device, (b) instrument, and (c) tissue excision quantity vs. plasma exposure time.

the current in the body to an extremely low level in comparison with that with the APC configuration. The system employs discharge plasmas in solutions instead of atmospheric needle-type plasmas, for surgical therapy and procedures [9]. A high frequency $(100 \mathrm{kHz})$ square-wave voltage pulse is applied to a highly electroconductive solution such as physiological saline, and the instrument thus embodies a repeatedly-pulsed discharge plasma configuration (Fig. 6(a)). When the applied voltage exceeds the threshold value, bubble clusters of several hundred micrometers diameter are generated together with the plasma light emission. When the voltage is further increased, breakdown occurs in the vapor layer and the plasma is generated (density $\sim 10^{12} \mathrm{~cm}^{-3}$, electron temperature $\sim 4 \mathrm{eV}$ ). Various types of instruments have already been developed (e.g., Fig. 6(b)), and as shown in Fig. 6 (c), it has been demonstrated that the mass of the surgically excised tissue (bovine myocardium) increases linearly with the plasma exposure time. Optical emission spectroscopy has shown the presence of $\mathrm{OH}$ and $\mathrm{H}$ radicals in the plasma, indicating that the mechanism of the plasma effect on the tissue involves etching of the tissue by $\mathrm{OH}$ radicals, and thus presumably a chemical mechanism of fibrous-protein collagen fragmentation.

\section{Next-Generation Biomedical Appli- cations of Nanocarbons}

We next discuss the current state of cutting edge research on nanocarbon intracellular transport and drug delivery systems, and the challenges still faced. Among the various nanocarbons, carbon nanotubes (CNTs) in particular are being investigated for in vivo transport, in parallel with gold nanoparticles. CNTs in unmodified form are

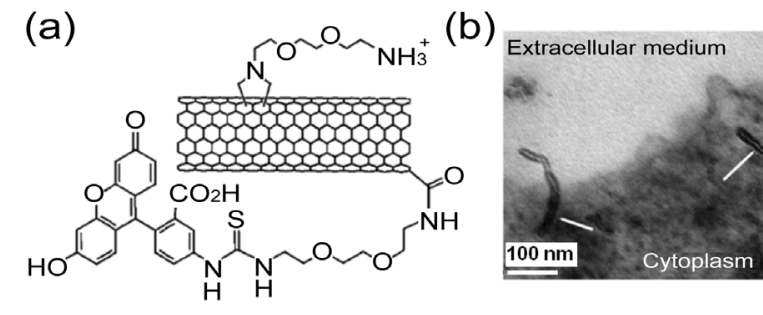

(c)

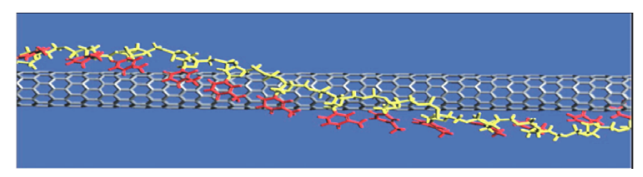

Fig. 7 (a) Carbon nanotube modified by aqueous ammonia and labeled with fluorescent compound, (b) cell membrane crossing of CNTs, and (c) SWNT wrapped with DNA.

hydrophobic and have low biocompatibility, and they are therefore synthesized in the form of water-soluble singlewalled carbon nanotubes (SWNTs) with side-wall chemical modification by amino-acid derivatives. A strongly fluorescent-luminescence portion is formed by addition of fluorescein isothiocyanate (FITC) (Fig. 7 (a)), to enable observation by confocal microscopy for tracking the in vivo SWNT. It has been demonstrated that when such labeled SWNTs are introduced into the body, they can penetrate cell membranes in the manner of "nanoneedles" which are absorbed and taken up by mammalian cells without cell lethality (Fig. 7(b)) and are in part excreted via the urine [10].

Technology has been developed for utilization of the intrinsic bandgap-origin photoluminescence of these SWNTs [11] for long-term continuous mapping of their transport through the body in a process termed singleparticle tracking (SPT) [12]. Figure 7 (c) shows a SWNT, the outside surface of which is wrapped with DNA having a hydrophilic portion (DNA-SWNT). The micrographs in Fig. 8 present the process of DNA-SWNT transport into and out of an NIH-3T3 cell. The cell is illuminated by a halogen lamp, and the DNA-SWNT is excited by a nearinfrared wavelength laser to stimulate light emission and thus enable its tracking. The micrographs show the adsorption of the DNA-SWNT on the cell membrane and its traversal in the endocytosis step, its confined-diffusion motion on the cell membrane after internalization, and the exocytosis and desorption step, thus encompassing the complete orbit of SWNT in vivo behavior. This achievement holds important implications for utilization of nanomaterials as cell sensors and other applications to biological systems. It has also been shown that the SWNT exocytosis and endocytosis rates mutually balance, in effect forming a self-regulating mechanism that prevents SWNT accumulation, thus maintaining the intracellular concentration below cytotoxic levels. This explains the apparent absence of cytotoxicity observed for many nanoparticle systems even 

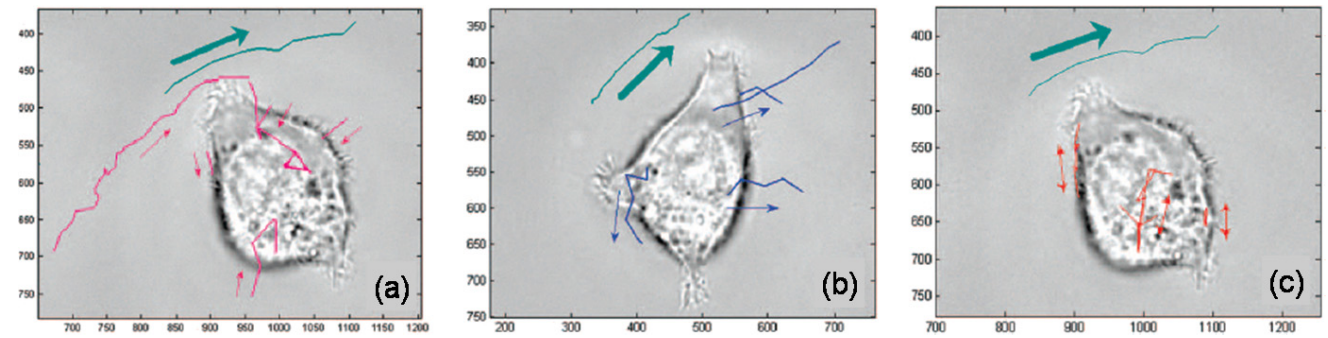

Fig. 8 DNA-SWNT intra- and extra-cellular transport tracking; (a) endocytosis, (b) excytosis, (c) intra-cell and cell-membrane diffusion.

under long-term exposure. It has been found, moreover, that the DNA-SWNT length resulting in the maximum cellular uptake efficiency is $320 \mathrm{~nm}$, which corresponds to an effective capture radius of $24.6 \mathrm{~nm}$ and is thus very close to the radius of $25 \mathrm{~nm}$ for maximum-efficiency uptake of gold nanoparticles.

As a nanomaterial candidate for applications in drug delivery systems (DDSs), CNTs are expected to enable simultaneous diagnosis, transport, and targeted delivery. In potential applications for gene delivery, the potency of supramolecular complexes, in which the CNT outside wall is functionalized by chemical modification using antibiotics, anticancer agents, and cell antigens, is gradually being elucidated, and CNTs are therefore expected to provide an effective means of infectious disease prevention, tumor therapy, and development of synthetic vaccines [10]. As a key application in genomic research, it is expected that the above-described DNA-SWNT complexes will be specialized for in vivo intracellular delivery, with the DNA as a probe for monitoring concentrations and particle counts of intracellular molecules and ions. The use of DNASWNTs may resolve a key problem that has impeded delivery by DNA alone, which is its susceptibility to degradation and its reduced efficacy in in vivo transport by the combined effects of nuclease digestion and enwrapment binding by single-stranded proteins. With DNA-SWNTs, in contrast, the bound DNA cargo is protected from enzymatic cleavage and binding protein interference. The selfdelivery capability and intracellular biostability of DNASWNT when targeting a specific ribonucleic acid (mRNA) cells in vivo are clearly far higher than those of independent DNA probes [13]. Its resistance to loss of potency due to cellular activity also makes it highly promising for therapeutic applications, as it is expected to enable DNA-based drugs to exert their therapeutic effects over longer periods before degradation.

The single-walled carbon nanohorn (SWNH) is also a highly promising configuration for targeted drug delivery. With a larger diameter $(2 \sim 5 \mathrm{~nm})$ than the SWNT configuration (1 2 nm) and a conical tip (Fig. 9, TEM image), it facilitates drug encapsulation and extended release. SWNHox, a SWNH with holes pre-formed in its walls by oxidation processing, has shown promising results in in vivo experiments with rats bearing transplanted tumors. When

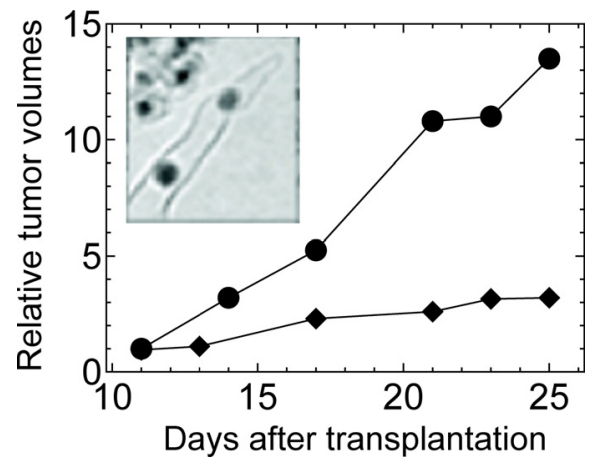

Fig. 9 Effect of SWNT systems for drug delivery (DDS) to transplanted mouse tumor; tumor volume vs. number of days after drug administration ( cisplatin (CDDP); • CDDP@SWNH $\mathrm{OX}$ ). Insert shows TEM image of encapsulated nanohorn.

used to encapsulate the anticancer agent cisplatin (CDDP) and injected directly into the tumors as CDDP@SWNHox, it has been found to suppress tumor growth far more effectively than CDDP alone (Fig. 9), presumably because of its gradual in vivo delivery of CDDP in high local concentrations in the tissue and efficient attack on the tumor cells [14].

The utilization of fullerenes [15] for in vivo transport also holds promise for applications in medical treatment and pharmaceuticals. In treatment for prevention of in vivo DNA damage and skin aging, fullerenes demonstrate the highest level of singlet oxygen removal capability. By inhibiting HIV protease, they interrupt the HIV growth cycle, and their development as an anti-AIDS drug is in progress. They are also promising as a therapeutic agent for amyotrophic lateral sclerosis. In relation to fullerenes with encapsulated atoms in their hollow internal spaces, referred to as "atom encapsulated (@) fullerenes", interest is particularly high concerning gadolinium encapsulated fullerene $\left(\left(\mathrm{Gd} @ \mathrm{C}_{82}\right)\right.$; used in the water-soluble form $\left.\mathrm{Gd} @ \mathrm{C}_{82}(\mathrm{OH})_{40}\right)$ as a contrast agent in nuclear magnetic resonance imaging (MRI). As illustrated in Fig. 10, its imaging effect in in vivo experiments with lung, liver, spleen, and kidney administration has been found nearly 20 times as strong as that of conventional contrast agents [16]. 


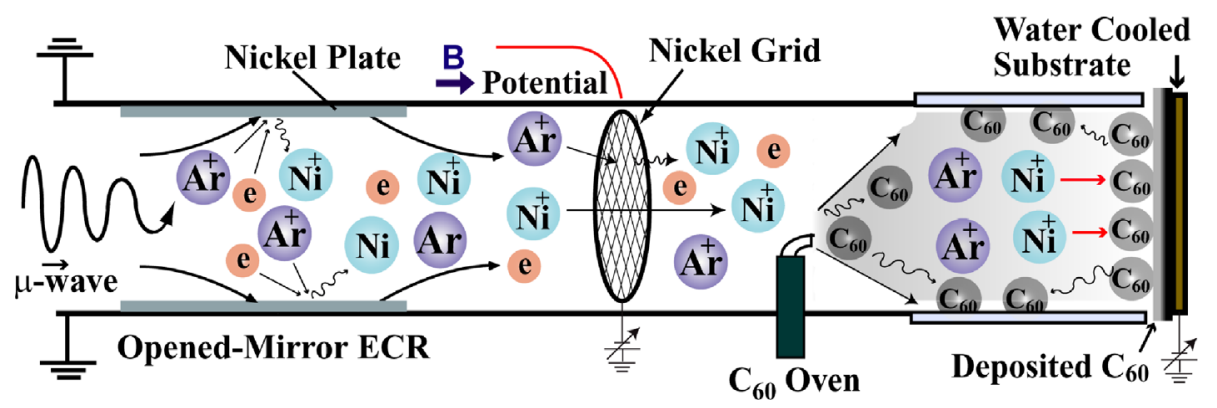

Fig. 11 Synthesis of ferromagnetic-metal atom encapsulated $\mathrm{C}_{60}$ by ECR plasma hybrid sputtering.

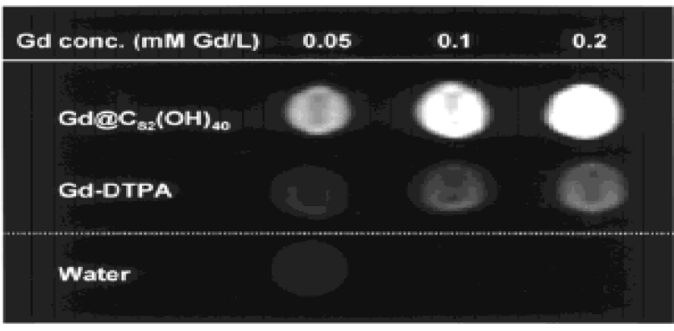

Fig. 10 MRI signal intensification ratios with atom encapsulated fullerene $\mathrm{Gd} @ \mathrm{C}_{82}(\mathrm{OH})_{40}$ and with conventional Gd-DTPA (water shown as reference).

\section{The Developmental Pathway from Plasma Nanobio Processes to Plasma Nanobioelectronics}

Nanocarbons hold great promise for many phases of advanced biological and medical applications. SWNTs, with their inherent luminescence capabilities, can provide superior in vivo imaging effects without addition of fluorescent labeling compounds. Double-walled carbon nanotubes (DWNTs) are furnished with their large internal spaces compared with SWNTs. The exploitation of inner nanospaces of SWNTs and DWNTs is of particular importance for T-cell and primary cell delivery while avoiding the microscopic obstacles of nuclease digestion and protein binding and enwrapment. Similarly at the cutting edge are the complementary and synergistic effects of gold nanoparticles and SWNTs/DWNTs, and the utilization of the inner nanospaces of fullerenes. For all of these nanomaterials, it is highly desirable to elucidate the latent potentials for biomedical applications. The following is a research activity currently being conducted by the authors for this purpose in relation to nanocarbons-based biological and medical applications utilizing gas phase, liquid phase, liquidsolid interfacial, and gas-liquid interfacial plasmas.

\subsection{Gas-phase plasma mediated fullerene nanobioelectronics}

As briefly described in Sec. 3 above, the application fields currently most promising for fullerenes are medicine and pharmaceuticals [15], and atom encapsulated fullerenes hold the potential to raise their effectiveness to a higher level. At present, however, the synthesized atom encapsulated fullerenes are chiefly not in the form of $\mathrm{C}_{60}$, the productivity of which is the largest in the empty state, but rather in the form of higher-order fullerenes such as $\mathrm{C}_{82}$ and $\mathrm{C}_{84}$, which are produced in quantities some two orders of magnitude lower than that of $\mathrm{C}_{60}$. Generally, the mass synthesis of atom encapsulated fullerenes has proven quite impossible, and even their effective synthesis in any quantity has been extremely difficult. The authors have therefore pursued the synthesis of "atom@ $\mathrm{C}_{60}$ ", and have very recently succeeded in the world's first large-volume synthesis of charge-exploited Li@ $\mathrm{C}_{60}$, using an original ion-irradiation method under the control of alkali-metal plasmas. We have also been able, by thorough parameter optimization in an electron-beam generation device with grid-separated double plasmas consisting of plasma generation and process regions, to achieve high-efficiency nitrogen atom dissociation and ionization [17], and to raise the synthesis purity of spin-exploited $\mathrm{N} @ \mathrm{C}_{60}$ to the world's highest level.

Ferromagnetic-magnetic atom encapsulated fullerenes are capable of both charge and spin exploitation but they have not yet been synthesized by the usual arc discharge methods, which are generally ineffective for this purpose because of their high surface tension with carbon and magnetic atoms. Experiments by the authors are now in progress for the world's first synthesis of $\mathrm{Ni} @ \mathrm{C}_{60}$. In this synthesis process (Fig. 11), a Ni plate is placed in a magnetic-mirror well and a $\mathrm{Ni}$ control grid is installed at the interface between the plasma generation and process regions, and synergistic sputtering from them is successfully carried out by an ECR argon plasma. The Ni atoms or ions are thus efficiently generated by control of the ion energy, and are externally injected into $\mathrm{C}_{60}$ particles from the outside. $\mathrm{Ni} @ \mathrm{C}_{60}$ is of interest as a novel magnetic semiconductor, and its high sensitivity to magnetic and electric fields is expected to lead to applications in diagnostics, imaging, and therapies such as a contrast agent in nuclear magnetic resonance imaging and hyperthermal therapy using microwaves and AC magnetic fields. 


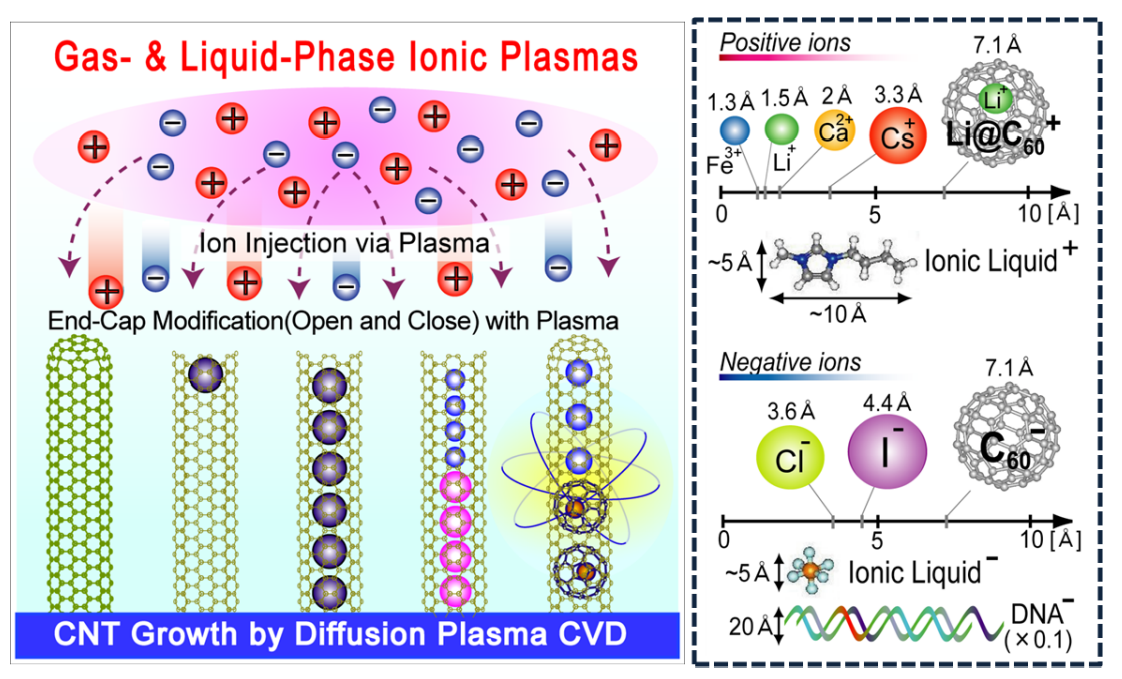

Fig. 12 Plasma system for nanospace control based on individual freestanding CNTs, as a form of nanoscopic plasma processing.

\subsection{Gas-phase plasma mediated nanotube nanoprocesses}

It is rather easy to functionalize SWNTs and DWNTs by chemical modification of their external walls, compared with the case where their internal spaces are exploited as nanocontainers, and this is currently the case in research on diagnostic and therapeutic applications as described in Sec. 3.

We have proposed a novel process (Fig. 12) in which pristine (empty) CNTs are grown in the individually freestanding form by plasma-enhanced chemical vapor deposition (CVD) [18] and ions in different kind and polarity ion plasmas separately generated are injected through their tips opened simultaneously, which are then closed. The process is thus an integrated form of nanoscopic plasma processing [19]. In this case, electronic interaction is promoted between the encapsulated atoms and/or molecules and the inner walls of the CNTs [20], thus creating CNTs which can manifest new properties, while it has also been necessary to develop plasmas composed of positive and negative ions with electron donor and acceptor abilities appropriate for this purpose. In the gas phase plasma thus generated, it has been possible to encapsulate (@) various molecules and atoms inside the CNT nanospace through selective acceleration of positive and negative ions in gas-solid interfacial sheath electric fields which are formed by applying DC bias voltages to CNTs-precoated substrate electrodes introduced into the plasmas. Figure 13 shows TEM images of three typical examples: $\mathrm{C}_{60} @$ SWNT [21], obtained with application of a positive bias; Cs@SWNT [22], obtained with application of a negative bias; and $\left(\mathrm{Cs} / \mathrm{C}_{60}\right) @ \mathrm{SWNT}$ (pn-junction housing nanotube) [23] obtained with application of polarity-reversed positive and negative biases.

As described in the following section, the positive and negative ion sources may consist of not only atoms and molecules for gas phase plasmas, but also biological polymer DNA and ionic liquids for liquid phase plasmas which
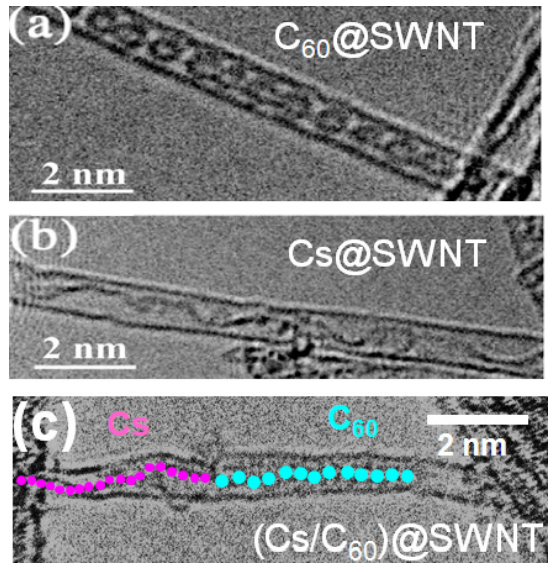

Fig. 13 TEM images of SWNTs with: (a) molecule encapsulation, (b) atom encapsulation, and (c) atom/molecule junction encapsulation.

are advantageous for nanobio processes.

\subsection{Liquid-phase mediated nanotube nano- processes}

In the light of the above, the authors envisioned the possibility of obtaining CNT-DNA conjugates, consisting of CNTs with DNA or other biomolecules encapsulated in their inner spaces. With DNA existing as negative ions against the background of counter positive ions in solutions, and taking the DNA solution as an "electrolyte plasma" [24], we applied the substrate bias method described above for gas phase plasmas to this liquid phase plasma (Fig. 14(a)). As the DNA in the solution assumes a large-diameter random-coiled shape (Fig. 14(b)), to facilitate its encapsulation in the smalldiameter $(\sim 1.4 \mathrm{~nm})$ SWNTs by drawing it into an elongated state through the effect of a high-frequency electric 


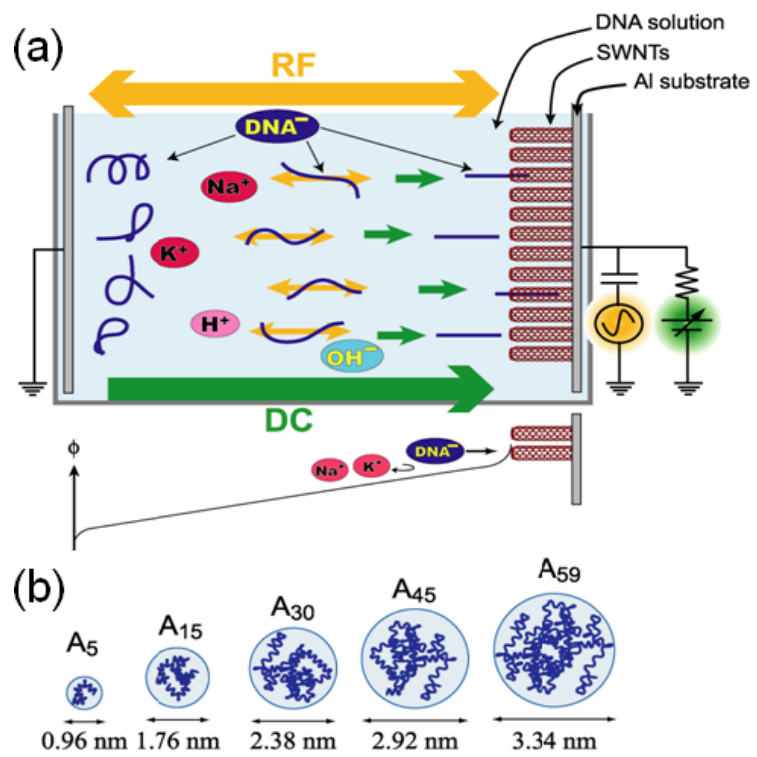

Fig. 14 (a) Process schematic of substrate bias method with electrolyte plasma in DNA solution, and (b) random-coiled single-stranded DNA (adenine: A) showing relation between number of bases (e.g., $\mathrm{A}_{30}$ ) and effective diameter.

(a)

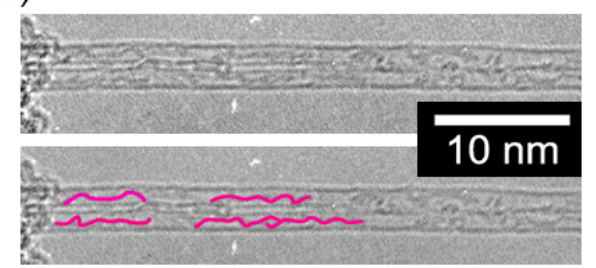

(b)

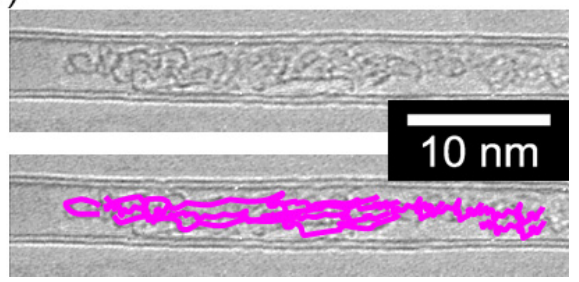

Fig. 15 TEM images demonstrating: (a) adenine DNA $\left(\mathrm{A}_{15}\right)$ encapsulated SWNT and (b) cytosine DNA $\left(\mathrm{C}_{30}\right)$ encapsulated DWNT. The encapsulation is outlined schematically by red lines.

field [25], we applied a positive bias for selective acceleration of the negative ions toward the CNTs-coated substrate and superimposed a high-frequency voltage upon it. In this way, we succeeded in injecting the DNA negative ions into the CNT internal spaces via the liquidsolid interfacial double layer [26] and thus in the creation of the world's first "single-stranded DNA@SWNT", "single-stranded DNA@DWNT", and "double-stranded DNA@DWNT" as confirmed by Raman scattering spectroscopy and TEM observation (Fig. 15).

In biosensor applications, this DNA encapsulation, in

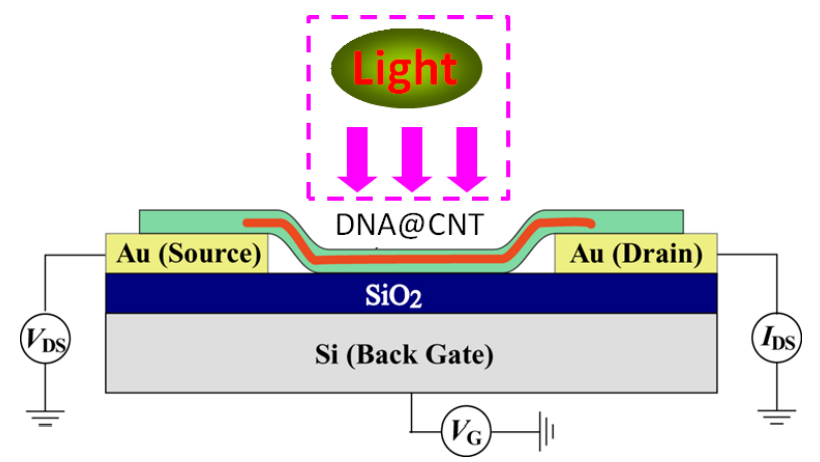

Fig. 16 FET with DNA encapsulated CNT (dotted-line insert: optional light illumination).

contrast to peripherally-wrapped CNT configurations, is reusable, and in advanced DDS applications it will enable cellular absorption and uptake without degradation of its functions during in vivo transport. When our attention is directed toward in vivo intracellular delivery, it also holds the promise of applications as a monitoring probe in genomic research, and as an intracellular nanoengineering tool for new cellular phenotype expression and for novel cellular functions and activities.

\subsection{Carbon nanotube nanobioelectronics}

In single-stranded (ss) DNA, the four bases adenine (A), guainine $(\mathrm{G})$, cytosine $(\mathrm{C})$, and thymine $(\mathrm{T})$ differ in oxidation and reduction potentiasl and in ionization potential [27]. If they are encapsulated in CNTs, characteristic differences therefore arise in the state of their electronic interaction with the inside wall of the CNTs and thus in their charge transfer state, which may be expected to provide a basis for modification and control of the CNT electrical properties. We therefore investigated the semiconducting conductance of each of several DNA@CNTs created by the above-described method of substrate bias in the DNA electrolytic plasma, by positioning it as the current channel in a field effect transistor (FET) (as in Fig. 16, without light illumination), varying the gate voltage $V_{\mathrm{G}}$ and the source-drain voltage $V_{\mathrm{DS}}$, and measuring the source-drain current $I_{\mathrm{DS}}$. As a result, CssDNA@SWNT (SWNT encapsulating cytocine singlestranded DNA) remarkably enhanced p-type conductance compared with the pristine/empty SWNT, and, in contrast, GssDNA@SWNT drastically showed n-type semiconductor conductance (Fig. 17 (a)) [28]. Similarly, although empty DWNT (inner diameter $\sim 4.0 \mathrm{~nm}$ ), because of its narrow band-gap, is an ambipolar conductive semiconductor simultaneously acting as both a p-type and an n-type conductance semiconductor, CssDNA@DWNT behaved as a monopolar p-type semiconductor and GssDNA@DWNT behaved as a monopolar n-type semiconductor [29].

To elucidate these changes in electrical properties, we 
(a)

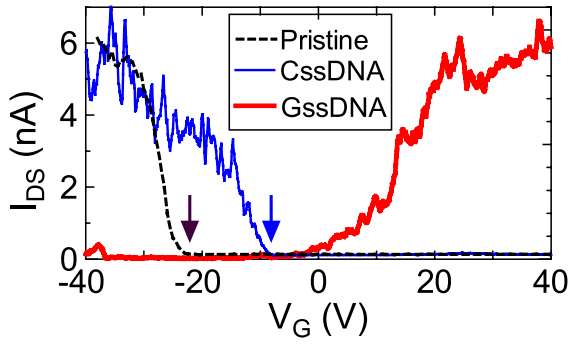

(b)

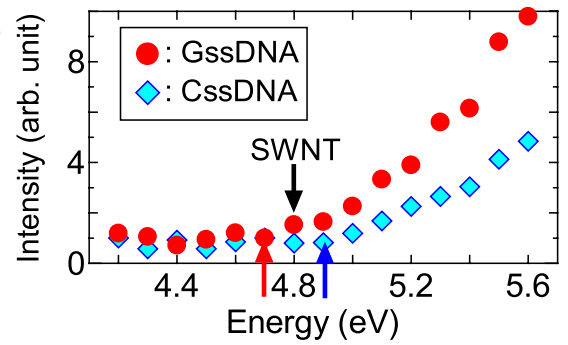

Fig. 17 (a) Electrical properties of DNA encapsulated SWNT: without illumination (dotted line), CssDNA encapsulation (thin line), and GssDNA encapsulation (thick line). (b) Ultraviolet photoemission spectra of DNA encapsulated SWNT: CssDNA (•), GssDNA (•), SWNT ionization potential (upper arrow).

measured the ionizing potentials of CNT and the DNA bases by ultraviolet photoelectron spectroscopy, and found the ionization potential of guainine to be approximately $0.2 \mathrm{eV}$ smaller than that of cytocine, and that of the SWNT to be intermediate between the two (Fig. 17 (b)), suggesting that guanine acts as an electron donor to the SWNT and cytosine as an electron acceptor, resulting in electrical characterization of SWNT as an n-type semiconductor and strengthening of its p-type character, respectively. In this way, it was confirmed that the choice of DNA base can control the CNT electrical properties and that the appropriate combination of bases enables the formation of a pn-junction configuration.

This has led to the world's first discovery of photoinduced electron transfer in which the CNT-DNA conjugate, in response to light illumination in the UV-vis range, undergoes a threshold-voltage shift in its FET transfer characteristics [30]. In the case of Gss@DWNT, the $V_{\mathrm{G}}-$ $I_{\mathrm{DS}}$ characteristic shifts in the direction of n-type enhancement ( $V_{\mathrm{G}}$ in the negative direction), and returns to the previous state when the illumination stops, thus clearly showing reversibility (Fig. 18). This is induced by the transfer of the DNA electrons to the CNT under excitation by the light illumination, and it holds the promise of application development for DNA@CNT organic semiconductors as photoswitching nanobio devices. The formation of quantum dots at low temperatures has also been verified [31], and we are pursuing the possibilities that these unique properties of nanobio-electronics devices endow biosensing and DDS systems with innovative functions.

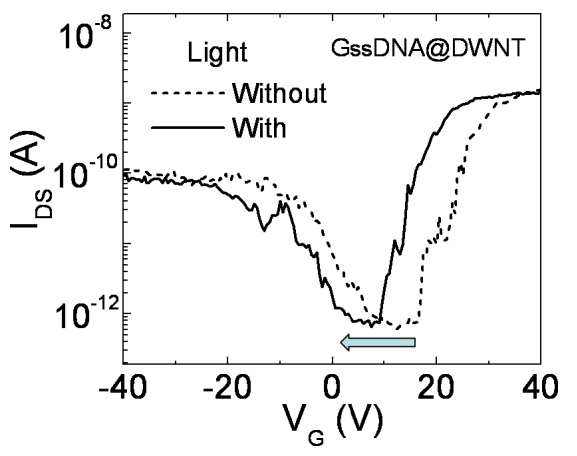

Fig. 18 Effect of light illumination on electrical properties of DNA encapsulated CNT.

\subsection{Gas-liquid interfacial plasma nanobio processes toward nanobiotronics}

With their size of several to several tens of nanometers, nanoparticles hold the key to development of in vivo DDS with protective functions, plasmonic treatment of cancer, and other nanomedical applications. Here, we take up gas- and liquid-phase interfacial plasmas sandwiched between gaseous plasmas and liquids, which represent key new reaction sites for the synthesis of gold nanoparticles significant for the immobilization of DNA and so on and for the creation of CNT-nanoparticle-DNA conjugates. Furthermore, we make mention of the related biological and medical applications. For the formation of a stable gasliquid interface, the liquid must comprise only positive and negative ions with no presence of water or any other solvent (thus, a fully- ionized liquid plasma), and must be in the liquid state at room temperature. This is called "ionic liquid" [32], which we adopt here. The ionic liquid itself is expected to find biological and medical applications in enzyme stabilization and activation, protein preservation solvents, metalloprotein synthesis, and antibacterial agents.

In this process, the DC voltage $V_{\mathrm{DC}}$ is applied to the "cathode electrode" located in the ionic liquid, causing an electrical discharge between it and the "anode electrode" located in the gas phase just above the surface of the ionic liquid, and enabling the generation of a stable gas-liquid interfacial plasma at both atmospheric and low pressures (Fig. 19 (a)) [33,34]. Measurements of space potential profiles with a Langmuir probe show the formation of a sheath electric field or an electrical double layer, and the presence of a large electrostatic electric field which accelerates the argon or other gas plasma ions in the direction of the liquid (the "ion irradiation mode"; Fig. 20 (a)). If, on the other hand, the electrode in the ionic liquid is used as the anode, an electrostatic electric field having the reverse polarity is formed, and the electrons in the gas-phase plasma are accelerated in the direction of the ionic liquid (the "electron shower mode"; Fig. 20 (b)).

If a gold compound (chlorauric acid trihydrate: $\mathrm{HAuCl}_{4} \cdot 3 \mathrm{H}_{2} \mathrm{O}$ ) is dissolved in the ionic liquid in advance, 
then with either the ion irradiation or the electron shower from the gas-phase plasma, gold nanoparticles are synthesized by reduction of the gold ions (Fig. 21) [35, 36]. It has been found that the ion irradiation induces the gener-

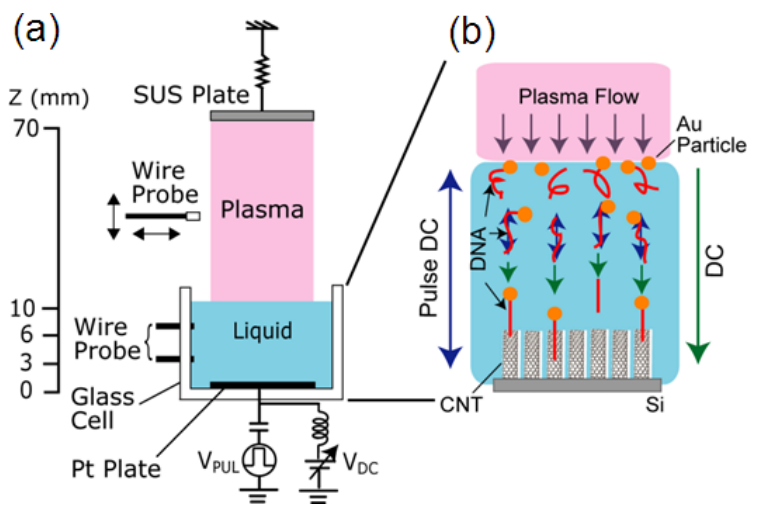

Fig. 19 (a) Gas-liquid interfacial plasma experimental apparatus and (b) CNT-nanoparticle-DNA conjugate creation process. (a)

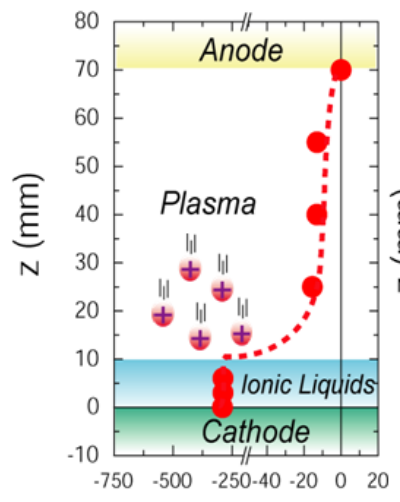

Potential (V)

\section{(b)}

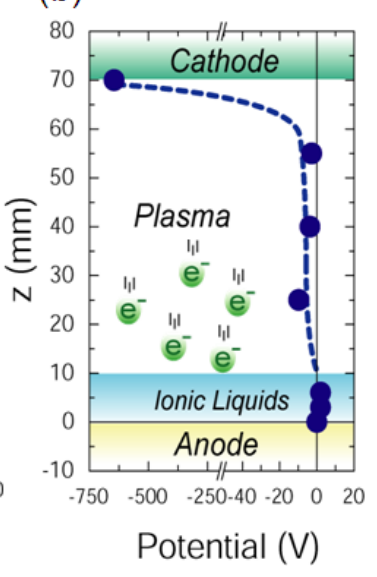

Fig. 20 Plasma space potential profiles extending from gas- to liquid-phase regions: (a) with liquid-phase electrode as cathode and (b) with gas-phase electrode as cathode. ation of hydrogen radicals in the ionic liquid with a reduction activity superior to that of electrons, thus resulting in higher synthesis-process efficiency. It has furthermore been found that if CNT is first dispersed in the ionic liquid as a nanotemplate and a gold compound is allowed to permeate throughout the liquid, then upon plasma irradiation from the gas phase the interstitial nanospaces between the bundled SWNT layers form the synthesis field and gold nanoparticles with a uniform size (in the neighborhood of $2 \mathrm{~nm}$ ) are observed at high density between the SWNT layers (Fig. 22) [37]. This SWNT_-gold nanoparticle conjugate may be appropriate for applications in high-sensitivity protein sensing and other fields [38].

For applications such as high-sensitivity biosensing using surface plasmon resonance and optically manipulable DDS, the nanoparticle-DNA conjugates are tried to be first synthesized and then encapsulated in CNTs. It has been found that when gold nanoparticles are synthesized in the liquid phase by the above-described plasma reduction method and simultaneously reacted with DNA present in the solution to produce the conjugate, the aqueous solution turns a deep purplish-red as the DNA concentration increases and the absorbance intensity increases at $550 \mathrm{~nm}$, which indicates the quantity of synthesized gold nanoparticles. (Fig. 23 (a), (b)). In the experiment shown schematically in Fig. 23 (c), performed with the objective of injecting this gold nanoparticle-DNA conjugate into CNT
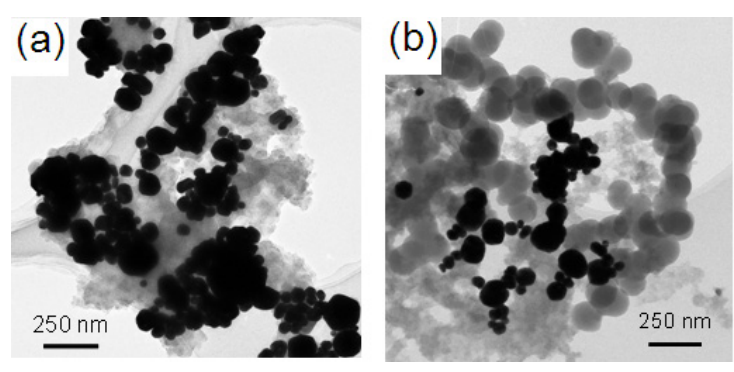

Fig. 21 TEM images of gold nanoparticles synthesized in (a) ion irradiation mode and (b) electron shower mode.
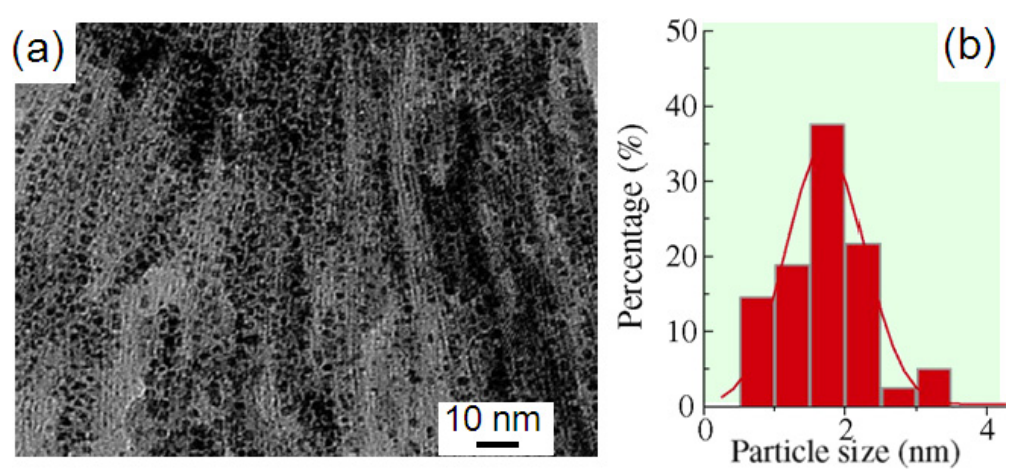

Fig. 22 (a) TEM image of SWNT_-gold nanoparticle conjugate synthesized with plasma irradiation and (b) gold nanoparticle size distribution. 
(a)

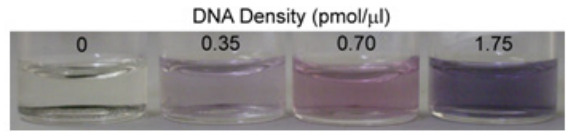

(b)

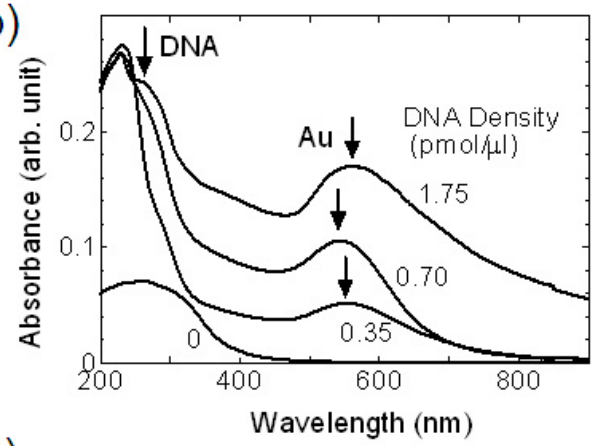

(c)

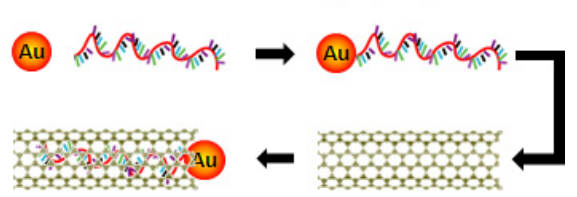

Fig. 23 (a) Photograph of aqueous solution of gold nanoparticles synthesized with plasma irradiation, (b) dependence of UV-visible light absorbance characteristics on DNA concentration, and (c) schematic of gold nanoparticle-DNA conjugate encapsulation in DNA.

as an embodiment of the process shown in Fig. 19, pulse DC voltages $V_{\text {pul }}$ superimposed upon positive DC voltages $V_{\text {DC }}$ were applied to the SWNT-coated electrode, and with the application of highly positive $V_{\mathrm{DC}}$ large quantities of the conjugate encapsulated in SWNT were observed for the first time. The remaining step for its application in advanced DDS is the development and establishment of a method for sustained release by optical manipulation of the gold nanoparticles in the conjugate to withdraw the encapsulated DNA from the CNT interior, and preliminary experiments for this purpose are now in progress.

\section{Summary}

We have discussed the current state of plasma processes in biological and medical applications including biocoating, inactivation, sterilization, disinfection, coagulation, therapy, and surgery, for the case of using lowand atmospheric-pressure plasmas generated by corona, glow, arc, and other discharge methods. Remarkable advances have been made in recent years in relation to threedimensional surface treatment by atmospheric-pressure plasma jet arrays, living tissue sterilization and therapy by floating-electrode dielectric barrier discharge plasmas, and surgical therapy and procedures by solution plasmas. Much uncertainty remains, however, concerning the physical and chemical effects and mechanisms of plasmas, and the advancement of fundamental research based on plasma diagnostics and measurements is essential.

We have also discussed next-generation biological and medical applications, focusing primarily on their interactions and composite effects between nanocarbons typefied by fullerenes (e.g., $\mathrm{C}_{60}$ ) and carbon nanotubes (CNTs) and biological polymers, and described the current state and cutting-edge challenges relating to intracellular transport and drug delivery systems.

From these observations, we have proceeded to a discussion of how plasmas open, create, and advance a nano-bio fusion science. More specifically, the development and utilization of advanced gas, liquid, and gas-liquid interfacial plasma processes have been practiced, realizing the creation of atom encapsulated $\mathrm{C}_{60}$ and biological-polymer encapsulated CNT based nanomaterials such as CNT - nanoparticle, nanoparticle-DNA and CNT_nanoparticle_-DNA conjugates, and the formation of their constituent devices, directed toward the development of biological and medical applications. We have discussed our approach, with these advances as the foundation, for the construction of plasma nanobio-electronics systems encompassing plasma nanobio devices, intracellular nanoengineering, and nanomedicine.

In closing, the authors would like to express our heartfelt gratitude to our collaborating researchers Assi. Prof. Toshiaki Kato, Assi. Prof. Yongfeng Li, Dr. Qiang Chen, and Mr. Takashi Harada.

[1] M. Inagaki and T. Kameyama, J. Plasma Fusion Res. 83, 595 (2007).

[2] N. Philip, B Saoudi, M.C. Crevier, M. Moisan, J. Barbeau and J. Pelletier, IEEE Trans. Plasma Science 30, 1429 (2002).

[3] M. Nagatsu, F. Terashita, H. Nonaka, L. Xu, T. Nagata and Y. Koide, Appl. Phys. Lett. 86, 211502 (2005).

[4] H. Eto, Y. Ono, A. Ogino and M. Nagatsu, Appl. Phys. Lett. 93, 221502 (2008).

[5] J.L. Walsh, J.J. Shi and M.G. Kong, Appl. Phys. Lett. 88, 171501 (2006).

[6] Z. Cao, J.L. Walsh and M.G. Kong, Appl. Phys. Lett. 94, 021501 (2009)

[7] G. Fridman, M. Peddinghaus, H. Ayan, A. Fridman, M. Balasubramanian, A. Gutsol, A. Brooks and G. Friedman, Plasma Chem. Plasma Process 26, 425 (2006).

[8] J. Raiser and M. Zenker, J. Phys. D: Appl. Phys. 39, 3520 (2006).

[9] K.R. Stalder, D. McMillen and J. Woloszko, J. Phys. D: Appl Phys. 38, 1728 (2005).

[10] M. Prato, K. Kostarelos and A. Bianco, Acc. Chem. Res. 41, 60 (2008)

[11] T. Kato and R. Hatakeyama, J. Am. Chem. Soc. 130, 8101 (2008).

[12] H. Jin, D.A. Heller and M.S. Strano, Nano Lett. 8, 1577 (2008).

[13] Y. Wu, J.A. Phillips, H. Liu, R. Yang and W. Tan, ACS Nano 2, 2023 (2008)

[14] K. Ajima, T. Murakami, Y. Mizoguchi, K. Tsuchida, T. Ichihashi, S. Iijima and M. Yudasaka, ACS Nano 2, 2057 (2008).

[15] K.M. Kadish and R.S. Ruoff Eds., Fullernes (John Wiley \& Sons, Inc., New York, 2000) p.437.

[16] M. Mikawa, H. Kato, M. Okumura, M. Narazaki, Y. 
Kanazawa, N. Miwa and H. Shinohara, Bioconjugate Chem. 12, 510 (2001).

[17] S. Miyanaga, T. Kaneko, H. Ishida and R. Hatakeyama, Thin Solid Films 518, 3509 (2010).

[18] T. Kato and R. Hatakeyama, Appl. Phys. Lett. 92, 031502 (2008).

[19] R. Hatakeyama, T. Kaneko, W. Oohara, Y.F. Li, T. Kato, K. Baba and J. Shishido, Plasma Sources Sci. Technol. 17, 024009 (2008).

[20] S.H. Kim, W.I. Choi, G. Kim, Y.J. Song, G.-H. Jeong, R. Hatakeyama, J. Ihm and Y. Kuk, Phy. Rev. Lett. 99, 256407 (2007).

[21] G.-H. Jeong, R. Hatakeyama, T. Hirata, K. Tohji, K. Motomiya, N. Sato and Y. Kawazoe, Appl. Phys. Lett. 79, 4213 (2001).

[22] G.-H. Jeong, A.A. Farajian, R. Hatakeyama, T. Hirata, T. Yaguchi, K. Tohji, H. Mizuseki and Y. Kawazoe, Phys. Rev. B 68, 075410 (2003).

[23] T. Kato, R. Hatakeyama, J. Shishido, W. Oohara and K. Tohji, Appl. Phys. Lett. 95, 083109 (2009).

[24] T. Kaneko, T. Okada and R. Hatakeyama, Contrib. Plasma Phys. 47, 57 (2007).

[25] M. Washizu and T.B. Jones, J. Electrostatics 38, 199 (1996).

[26] T. Okada, T. Kaneko, R. Hatakeyama and K. Tohji, Chem.
Phys. Lett. 417, 288 (2006).

[27] K.H. Yoo, D.H. Ha, J.O. Lee, J.W. Park, J. Kim, H.Y. Lee, T. Kawai and H.T. Choi, Phys. Rev. Lett. 87, 198102 (2001).

[28] T. Kaneko and R. Hatakeyama, Appl. Phys. Express 2, 127001 (2009).

[29] Y.F. Li, T. Kaneko and R. Hatakeyama, Small 6, 729 (2010).

[30] Y.F. Li, T. Kaneko, Y. Hirotsu and R. Hatakeyama, Small 6, 27 (2010).

[31] Y.F. Li, T. Kaneko and R. Hatakeyama, Appl. Phys. Lett. 96, 023104 (2010).

[32] K.R. Seddon, Nature Mater. 2, 363 (2003).

[33] K. Baba, T. Kaneko and R. Hatakeyama, Appl. Phys. Lett. 90, 201501 (2007).

[34] T. Kaneko, K. Baba and R. Hatakeyama, J. Appl. Phys. 105, 103306 (2009).

[35] K. Baba, T. Kaneko and R. Hatakeyama, Appl. Phys. Express 2, 035006 (2009).

[36] T. Kaneko, K. Baba, T. Harada and R. Hatakeyama, Plasma Process. Polym. 6, 713 (2009).

[37] K. Baba, T. Kaneko, R. Hatakeyama, K. Motomiya and K. Tohji, Chem. Commun. 46, 255 (2010).

[38] F.N. Ishikawa, B. Stauffer, D.A. Caron and C. Zhou, Bioisens. Bioelect. 24, 2967 (2009). 